of his problem and its distressing symptoms.

Failures do occur, as in every other kind of therapy, but any success in this "difficult" group is surely worthwhile, when hypnotherapy does turn out to have been the treatment of choice.

Gordon D K FLINT

Annan, Dumfriesshire

' British Medical fournal, 1978, 2, 978.

${ }^{3}$ Hartland, J H, Medical and Dental Hypnosis and its Clinical Applications. London, Baillière Tindall, 1977.

\section{Teratomas of the ovary}

SIR,-We think there are several important points that were not included in your leading article (21 April, p 1034) on teratomas of the ovary. Although rare, malignant teratomas of the ovary have many features in common with their testicular counterpart. In testicular teratomas the prognosis has improved considerably for three main reasons. Firstly, the majority of malignant teratomas are now known to produce either or both of the tumour markers human chorionic gonadotrophin (HCG) and $\alpha$-fetoprotein (AFP), which provide the most sensitive monitor of disease progression or regression. Secondly, new highly active cytotoxic drugs have been introduced, which are now used in combination. Thirdly, it has been recognised that bulky tumour deposits (greater than $5 \mathrm{~cm}$ diameter) may require surgical removal in order to obtain a complete clinical remission.

In our recent experience at Charing Cross Hospital we have treated eight ovarian teratomas with a cycling regimen of chemotherapy including platinum diamminochloride and etoposide (VP16-213), which is due to be reported shortly. The results were as follows. One patient died from malignant teratoma and one is still on treatment. Of the remaining six patients, five are off treatment (range 5-16 months), and one patient is well with a stable tumour, which has been biopsied on two occasions and both times showed differentiated teratoma. We would regard malignant ovarian teratomas as potentially curable tumours. The value of HCG and AFP in monitoring the treatment was confirmed and only one of the eight patients produced neither of these markers. Whole-body computerised tomography has also introduced a greater sensitivity in confirming complete clinical remissions.

Malignant ovarian tumours are rare and in order to combine the various treatment and diagnostic methods to get the optimum results these patients should be managed in a limited number of centres with a special interest in this tumour.

E S NEWLANDS R H J BEGENT G J S RUSTIN K D BagshaWE

Department of Medical Oncology, Charing Cross Hospital,
London W6 8RF

\section{Anaesthetic deaths and caesarean} section

SIR,-I regret that use of the head-down lateral position as advocated by $\mathrm{Dr} B \mathrm{H}$ Goodrich (21 April, p 1079) would not have prevented all the acid-aspiration syndromes that I have been asked to treat.

In two cases aspiration occured during recovery from anaesthesia and in one case this was undoubtedly preventable. This patient drank water about 1 hour before operation and after a straightforward $D$ and $C$ was returned to the recovery room unconscious lying on her back. The recovery nurses receiving her found the mouth full of clear fluid, which was being aspirated and subsequently induced a life-threatening aspiration syndrome.

I teach that all patients should be turned on their side and be in possession of protective reflexes before extubation. I also use a "therapeutic thump" to the lateral chest wall following extubation to clear any secretions, etc, from the upper airway where patients have had tonsillectomies, for instance.

Assuredly the hasty anaesthetist who extubates a half-paralysed patient while the houseman is still putting skin stitches in will find his enthusiasm tempered in the draughty corridors of the Queen's Bench.

Bromley, Kent

J M Cundy

\section{Treatment of onchocerciasis}

SIR,-Your expert answering a query about the treatment of onchocerciasis in your Any Questions? column (31 March, p 877) advised trying metrifonate as an alternative to diethylcarbamazine in an attempt to reduce the severity of the reaction caused by treatment.

The "reaction" is due to the release of antigens from the dead microfilariae, and recent controlled trials in the Onchocerciasis Chemotherapy Research Centre in Tamale, Ghana, have shown no advantage of metrifonate over diethylcarbamazine. Furthermore, the dosage advocated in the answer by your expert is not sufficient to remove microfilariae from the skin: it is only because it is less effective that this dose has slightly fewer side reactions.

The only rational management avoiding the use of suramin is to give an initial intensive course of treatment with diethylcarbamazine to rid the patient of the microfilariae from the skin, followed by weekly doses. The initial course almost invariably causes a reaction, and there is no way in which it can be avoided. It is wise to start with a small dose such as $50 \mathrm{mg}$ on the first day, $100 \mathrm{mg}$ on the second day, $200 \mathrm{mg}$ on the third day, $200 \mathrm{mg}$ twice daily on the fourth day, then the full dose of $200 \mathrm{mg}$ three times a day for three days. This regimen will kill almost all the microfilariae, and preliminary results suggest that an even shorter course may be equally effective. It is best to have the patient in hospital during this period because occasionally the reactions are severe, with fever, postural hypotension, and adenitis. Symptomatic relief can be obtained by using antihistamines, and the very severe reactions will occasionally require corticosteroids. If iritis develops, topical steroid drops should be used as well.

After ridding the patient of the microfilariae, the adult worms will remain alive, and if nothing further is done the skin will eventually be repopulated with microfilariae. In order to keep the microfilarial density in the skin low, without the need to give the potentially toxic drug suramin, diethylcarbamazine is given weekly. This drug is entirely without toxic effects and with such frequent administration the microfilariae never become sufficiently numerous to cause further reactions when the drug is taken. The initial cutaneous reaction to the dead microfilariae often takes the form of a chronic papular dermatosis, which may take three to four months to subside. The patient should be reassured about this, and the continuation of weekly dosage will ensure that the patient escapes further skin irritation and that the eyes do not become involved. Normally, a weekly dose of $200 \mathrm{mg}$ is quite sufficient; it should be continued for 10 years.

Incidentally, Simulium, the vector of onchocerciasis, is a black fly not a mosquito.

Liverpool School of

Tropical Medicine

DION BELL

\section{Enuresis and tricyclic antidepressants}

SIR,-Recent correspondence (7 April, p 951) on the subject of the use of tricyclic drugs in the treatment of enuresis illuminates one of the difficulties encountered by consultants and researchers when giving the benefit of their experiences to general practitioners-who are, in the main, the ones who have to contend with patients in their natural environment. I mean, of course, the difficulty of visualising the home conditions of the patient. At times the picture presented by some of your correspondents is idealised to the point of naivety.

In our group practice, which covers every type of family and home, the possession of adequate quantities of "easy care" linen and comprehensive laundry facilities is the exception: the family financial priorities put them rather low in the scale. Nor is the ability to produce hot water early in the morning for the necessary bathing all that common. The enuretic child may well share a bed; there may be siblings still at the napkin stage; there are often other enuretics in the family; and both parents may have to leave home early for work. The unfortunate child therefore goes to school with the faint but tell-tale smell around him and has to miss any social activities such as camps and visiting friends which entail spending a night away from home. Little wonder that there is great pressure for "something to be done" and that the practitioner, faced with waiting lists and bureaucracy when trying to arrange for a buzzer, prescribes the tricyclics that do help very many of the children.

The most splendid example of the unireality of our expectations lies in the advocacy of "childproof" containers, which are so difficult to open (except by children) that in most homes they are either left open or their contents decanted into another jar to form a marvellous pot-pourri of poisons.

\section{Sheffield S30 5GX}

\section{A OWEN GRIfFIthS}

\section{Poisoning with tricyclic} antidepressants

\section{SIR,-The report by Dr A J Cronin and others} (17 March, p 722) rightly emphasises the danger of overdose, but some of its premises are debatable. It may be true that "tricyclic antidepressants are now the commonest cause of fatal poisoning in children under the age of 5 years," but the figures they quote for the UK as a whole show a gross increase in such deaths from $3 \cdot 2$ annually in $1962-71$ to 3.4 annually in 1972-6-hardly a significant change. I agree that the buzzer "properly used" is 